\title{
Fractional anisotropy in white matter tracts of very-low-birth-weight infants
}

\author{
Jeroen Dudink • Maarten Lequin • Carola van Pul • \\ Jan Buijs • Nikk Conneman • \\ Johannes van Goudoever • Paul Govaert
}

Received: 25 April 2007 /Revised: 14 August 2007 / Accepted: 14 August 2007 / Published online: 2 October 2007

(C) Springer-Verlag 2007

\begin{abstract}
Background Advances in neonatal intensive care have not yet reduced the high incidence of neurodevelopmental disability among very-low-birth-weight (VLBW) infants. As neurological deficits are related to white-matter injury, early detection is important. Diffusion tensor imaging (DTI) could be an excellent tool for assessment of white-matter injury.

Objective To provide DTI fractional anisotropy (FA) reference values for white-matter tracts of VLBW infants for clinical use.

Materials and methods We retrospectively analysed DTI images of 28 VLBW infants (26-32 weeks gestational age) without evidence of white-matter abnormalities on conventional MRI sequences, and normal developmental outcome (assessed at age 1-3 years). For DTI an echoplanar sequence with diffusion gradient $\left(b=1,000 \mathrm{~s} / \mathrm{mm}^{2}\right)$ applied in 25 non-collinear directions was used. We measured FA
\end{abstract}

J. Dudink $(\bowtie) \cdot$ N. Conneman $\cdot$ J. van Goudoever $\cdot$ P. Govaert Division of Neonatology, Department of Paediatrics, Erasmus MC-Sophia Children's Hospital, P.O. Box 2060, 3000 CB Rotterdam, The Netherlands e-mail: j.dudink@erasmusmc.nl

\section{Lequin}

Division of Paediatrics, Department of Radiology, Erasmus MC-Sophia Children's Hospital,

Rotterdam, The Netherlands

C. van Pul

Department of Clinical Physics, Maxima Medical Center,

Veldhoven, The Netherlands

\section{J. Buijs}

Division of Neonatology, Department of Paediatrics,

Maxima Medical Center,

Veldhoven, The Netherlands and apparent diffusion coefficient (ADC) of different whitematter tracts in the first 4 days of life.

Results A statistically significant correlation was found between gestational age and FA of the posterior limb of the internal capsule in VLBW infants $(r=0.495, P<0.01)$.

Conclusion Values of FA and ADC were measured in white-matter tracts of VLBW infants. FA of the pyramidal tracts measured in the first few days after birth is related to gestational age.

Keywords Diffusion tensor imaging ·

Fractional anisotropy $\cdot$ MRI $\cdot$ Prematurity

\section{Introduction}

The last decade has seen improved survival for verylow-birth-weight (VLBW) preterm infants [1, 2]. The survivors, however, still show a high incidence of neurodevelopmental disability, despite advances in fetal and neonatal intensive care [3-5]. These neurological deficits are usually related to white-matter injury $[6,7]$. Thus early detection of abnormal white-matter maturation is important in the design of preventive, protective, and rehabilitative strategies for the management of the critically ill newborns $[8,9]$.

The clinical evaluation of these infants may not provide adequate diagnostic or prognostic information on whitematter injury. Various neuroimaging techniques have become available, however, that may be of help. Cranial US is such a technique used in premature infants, but it is not as sensitive as MR imaging, which shows better softtissue contrast [10]. Yet, conventional T1-weighted (T1-W) and T2-weighted (T2-W) MR imaging sequences also have limited value in the evaluation of the preterm brain, as they do not allow visualization of specific white-matter tracts 
before the onset of myelination. Most white-matter tracts of the premature cerebrum are unmyelinated except for a few pathways, e.g. the pyramidal tract that matures early as the infant approaches term-equivalent age [11, 12].

Diffusion tensor imaging (DTI) is unique in its ability to visualize and quantify white-matter tracts in the human brain. It is superior to T1-W and T2-W imaging in detecting unmyelinated or premyelinated fibre tracts [13] and, therefore, likewise in assessing the microstructural organization of the developing white matter. DTI anisotropy measurements offer great potential in the study of whitematter damage in VLBW infants. So far, however, the clinical use of this technique is restricted by the lack of normal reference values for VLBW infants. Although there are reports on fractional anisotropy (FA) and apparent diffusion coefficient (ADC) values in preterm infants, only four groups have reported values for infants of gestational age less than 32 weeks without brain injury (Table 1). Each of the populations studied included no more than four infants without white-matter damage on conventional imaging, scanned within days after birth and gestational age less than 32 weeks [14-17]. Because the sizes and shapes of the regions of interest (ROIs) in these studies differ, comparison between the studies is difficult. We therefore set up a retrospective study aimed at providing reference values for the clinical interpretation of DTI images in VLBW infants.

\section{Materials and methods}

\section{Subjects}

The Erasmus MC Ethical Review Board approved the study and written informed parental consent was obtained for each subject. The images used in this study had been obtained within the framework of a study in which premature infants of different gestational ages underwent serial conventional and DTI acquisitions to evaluate whitematter development. The inclusion criteria for our study were birth at gestational age 25-32 weeks, no evidence of white-matter injury on conventional MRI and scanned within 4 days of birth. Developmental outcome assessed at age 1-3 years needed to be normal. Gestational age was calculated from the mother's last menstrual period or estimated from early US ( $<18$ weeks of pregnancy). Exclusion criteria were intraventricular haemorrhage, ventriculomegaly, congenital infection, brain malformation or a multiple congenital anomaly syndrome. Furthermore, preterm infants whose images showed severe motion degradation were excluded since evaluation of these scans was not possible. During the 25 months of the study period (March 2004 to April 2006), 41 infants were scanned and 32 met our study criteria. Four MR examinations were excluded due to severe motion artefacts, so 28 patients were included.

\section{Neurodevelopment monitoring}

The infants' hospital charts were reviewed for neurodevelopmental outcome. They had all been neurologically assessed at 3, 6, 12 and 24 months, and Denver scores were used to monitor neurodevelopment [18]. At 24 months the Bayley scales of infant development, second edition (BSIDII) had also been applied [19]. All patients had shown normal neurodevelopment according to the most recent examination.

\section{Conventional MR imaging}

Images were acquired using standard scanning protocols. All imaging was performed on a 1.5-T GE EchoSpeed scanner (GE Medical Systems, Milwaukee, Wis.). The imaging protocol included T1-W (spin echo, TR/TE 500/ $11 \mathrm{~ms}$ ) and T2-W sequences (spin echo, TR/TE 3,000/ $120 \mathrm{~ms}$ ) with a slice thickness of $4 \mathrm{~mm}$ and $0.4 \mathrm{~mm}$ gap. For DTI an echoplanar sequence with diffusion gradients $\left(b=1,000 \mathrm{~s} / \mathrm{mm}^{2}\right)$ applied in 25 non-collinear directions was used with a slice thickness of $3 \mathrm{~mm}$ and no gap. An average of 20 slices was recorded within 4 min using TR/ TE 9,150/98-91 ms. The FOV was $20 \mathrm{~cm}$, the scan matrix $128 \times 128$ and the reconstruction matrix $256 \times 256$.

All patients were scanned using an MR-compatible incubator with a specialized high-sensitivity neonatal head coil (Lammers Medical Technology, Lubeck, Germany) that allowed DTI imaging at high spatial resolution and high signal-to-noise ratio (SNR). The incubator provided controlled temperature and humidity as well as MR-
Table 1 Overview of relevant literature

${ }^{a}$ ROI placement with fibre tracking.

\begin{tabular}{lllllll}
\hline Reference & $\begin{array}{l}\text { Gestational } \\
\text { age (weeks) }\end{array}$ & Scan week & $\begin{array}{l}\text { No. of } \\
\text { patients }\end{array}$ & $\begin{array}{l}\text { No. with gestational } \\
\text { age }<32 \text { weeks }\end{array}$ & $\begin{array}{l}\text { MR field } \\
\text { strength (T) }\end{array}$ & ROI \\
\hline$[14]$ & $<37$ & $28-43$ & 27 & $<5$ & 1.5 & FT $^{\mathrm{a}}$ \\
{$[15]$} & $24-36$ & $28-39$ & 17 & $<5$ & 1.5 & Manual \\
{$[16]$} & $24-36$ & $28-39$ & 14 & $<5$ & 1.5 & Manual \\
{$[17]$} & $26-30$ & $28-40$ & 6 & $<5$ & 1.5 & Manual \\
\hline
\end{tabular}


compatible pulse oximetry and ventilation. This set-up allowed imaging in this most vulnerable patient population in a stable and safe microenvironment [20-22]. We used mouldable earplugs and neonatal earmuffs to reduce the noise. No sedation was given. Pads around the infant's head kept movement to a minimum.

\section{Diffusion tensor postprocessing}

Diffusion tensor images were transferred to a GE Advantage Windows workstation (General Electric Medical Systems, Milwaukee, Wis.) for postprocessing using Functool 2000 software (General Electric). DTI measures the diffusion of water in each voxel and the extent to which water diffuses in particular directions as a result of the microstructural characteristics of the tissue imaged. High anisotropy indicates that the magnitude of diffusion is very unequal in different directions. The complex nature of anisotropic diffusion in the brain has been described by a diffusion tensor, which contains information about the magnitude of diffusion in different directions. Each tensor contains a set of three eigenvalues, which are related to the major, intermediate, and minor axes of a diffusion ellipsoid. The principal eigenvector $\left(\lambda_{1}\right)$ specifies the direction in which water diffusion is greatest. FA measures the fraction of the magnitude of the diffusion tensor that can be ascribed to anisotropic diffusion [23-25]. For isotropic diffusion $\left(\lambda_{1}=\lambda_{2}=\lambda_{3}\right)$, FA is zero, and in the case where there is a strongly preferred direction of diffusion $\left(\lambda_{1}>>\lambda_{2}=\lambda_{3}\right)$, FA approaches a value of one. The colour maps were based on major eigenvector orientation with red representing rightleft, green representing anteroposterior, and blue representing superoinferior anatomical directions.

The tracts selected for quantization in the study included commissural tracts (corpus callosum: splenium and genu), projection tracts including those of the posterior limb of the internal capsule (PLIC), the anterior limb of the internal capsule (ALIC) and the optic radiation (OR), and association tracts (external capsule, EC).

\section{Regions of interest}

DTI measurements were taken from multiple ROIs positioned bilaterally within individual white-matter tracts. For the placement we used standard-size, round-shaped 16-pixel ROIs. To optimize our ROI placement we first detected the patient scan slice that represented slice "O" of the atlas of Wakana et al. [26]. We then placed the ROI, combining the FA, ADC and colour map to verify the right location. When the two researchers both agreed on the position, the ROI was used as a seed point to perform fibre tracking (minimum length $10 \mathrm{~mm}$, minimum anisotropy
0.10). We compared the fibres that were visualized with the fibre bundles described in the atlas of Wakana et al. for verification (Figs. 1, 2 and 3). We then looked for the maximum pixel value within the ROI (Figs. 4 and 5). Two researchers experienced in neonatal DTI achieved consensus on ROI placement and measurements.

\section{Statistical analysis}

The relationship between FA of the white matter tracts and gestational age was analysed by correlation analysis (Pearson product moment correlation, SPSS 13.0.1). Tract comparison was done using one-way analysis of variance (ANOVA).

\section{Results}

Patient characteristics

Gestational ages at birth ranged from 26 to 32 weeks (mean 28 weeks 5 days). Mean weight at birth was $1,148 \mathrm{~g}$ and mean head circumference was $26.4 \mathrm{~cm}$. All patients had shown normal neurodevelopment as defined by the Denver or Bayley scoring system according to the most recent examination.

FA and ADC of ROIs of standard pixel size

We plotted the average FA and ADC values of 16-pixel ROIs of the different white-matter tracts against gestational

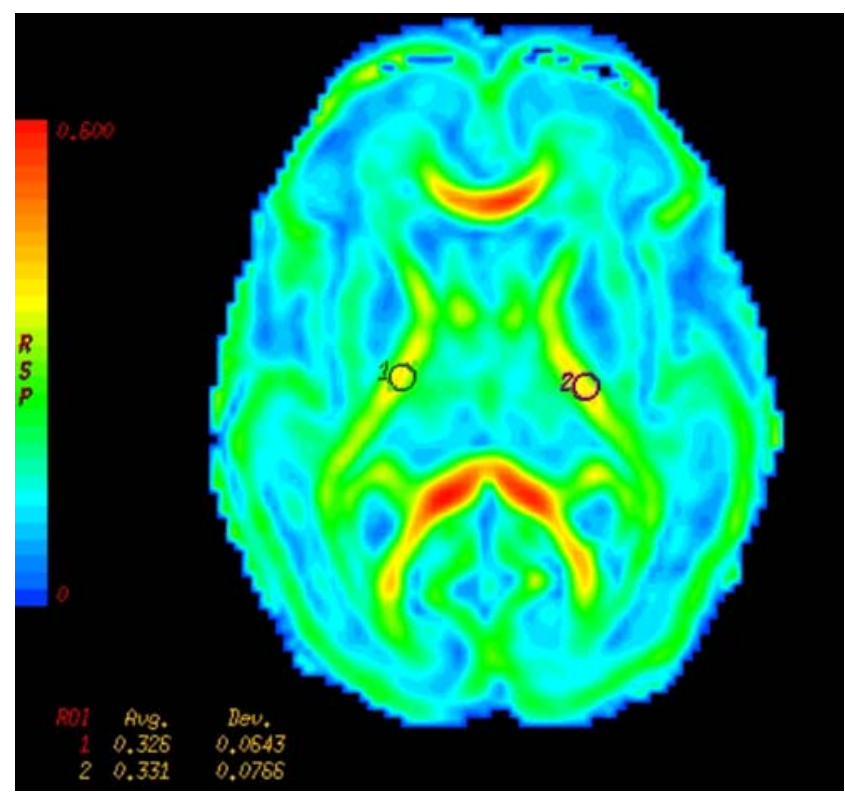

Fig. 1 Example of an FA map (gestational age 28 weeks) on which the ROIs are placed 


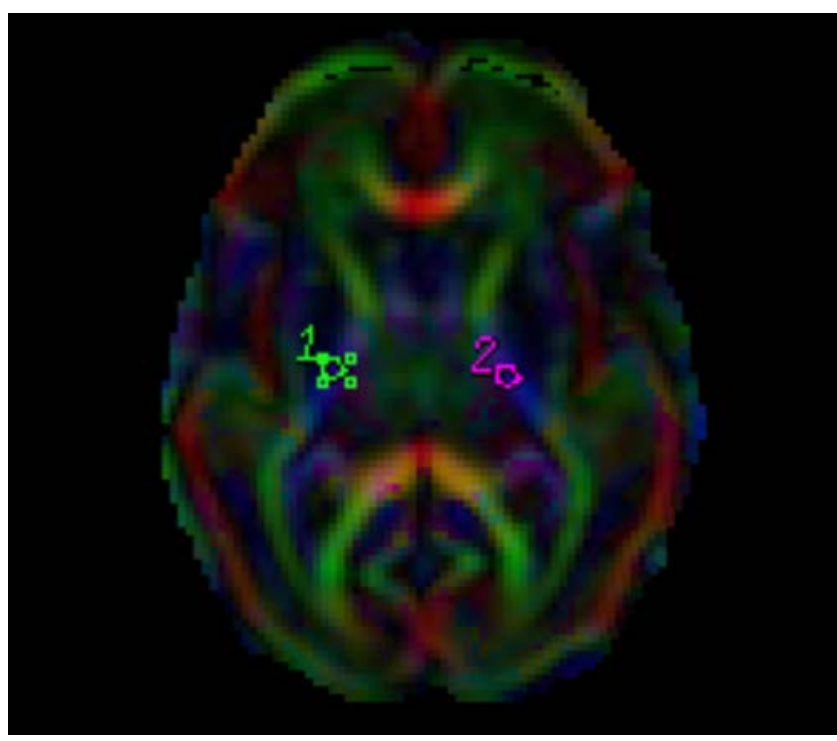

Fig. 2 Confirmation of ROI placement on the colour map (gestational age 28 weeks)

age. No statistically significant differences based on a twotailed paired Student's $t$-test (at a significance level of $P<0.05$ ) were found comparing FA and ADC values between the left and right hemispheres in each subject. Therefore, the values obtained from the left and right were averaged to obtain the mean FA and ADC of the different ROIs. We found a significant correlation between gestational age and FA of the PLIC ( $r=0.495, P<0.01$; Fig. 6). No significant correlations were found for the other tracts (Table 2). We found no correlation between average ADC values and gestational age in the studied tracts (Table 2).

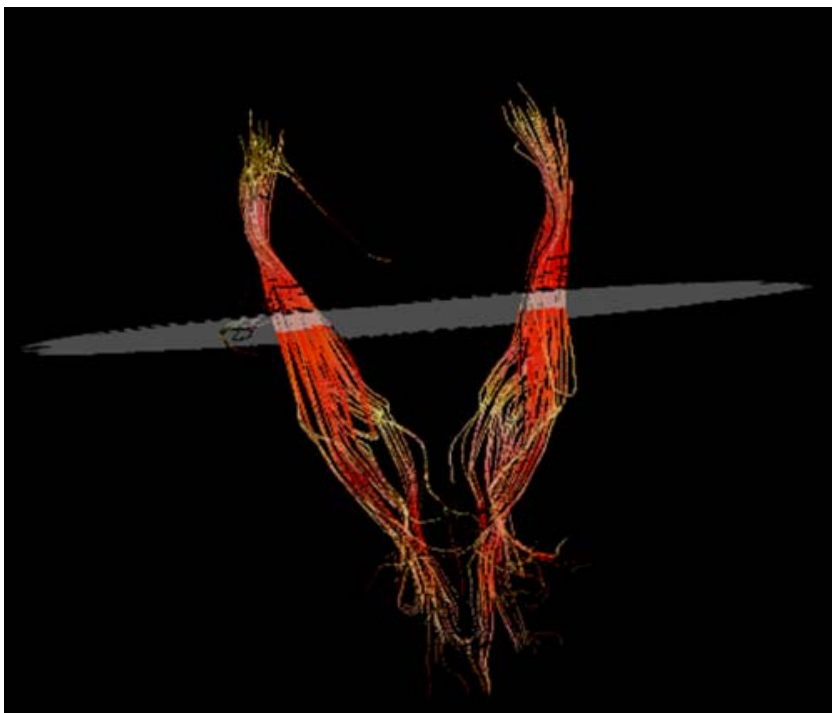

Fig. 3 Fibre tracking was performed in each of the ROIs to confirm the correct location. This is an example showing the corticospinal tracts (gestational age 28 weeks)

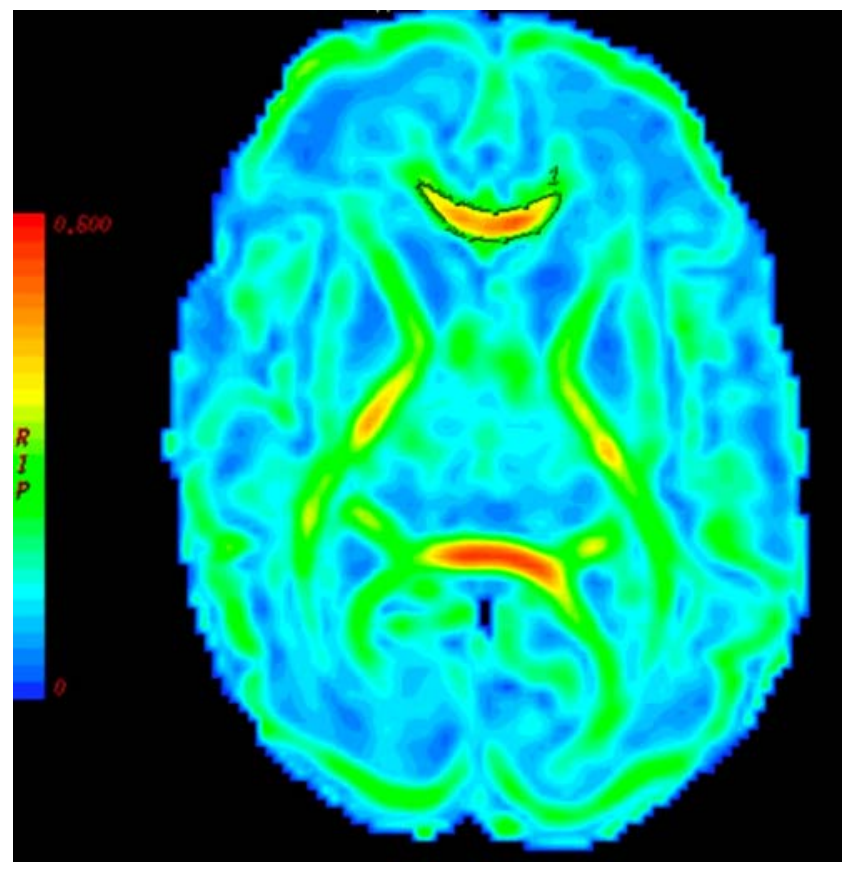

Fig. 4 Delineation of the corpus callosum using free-hand ROI placement on an FA map (gestational age 28 weeks)

Maximum FA values of the PLIC

The maximum FA values of the ROI were plotted against gestational age and also showed a significant correlation between gestational age and FA of the PLIC $(r=0.569$, $P<0.01$; Fig. 7). We saw no other significant correlations between FA and gestational age. Compared with standardsize ROI mean FA values of the PLIC, the maximum values were a mean of 0.05 higher.

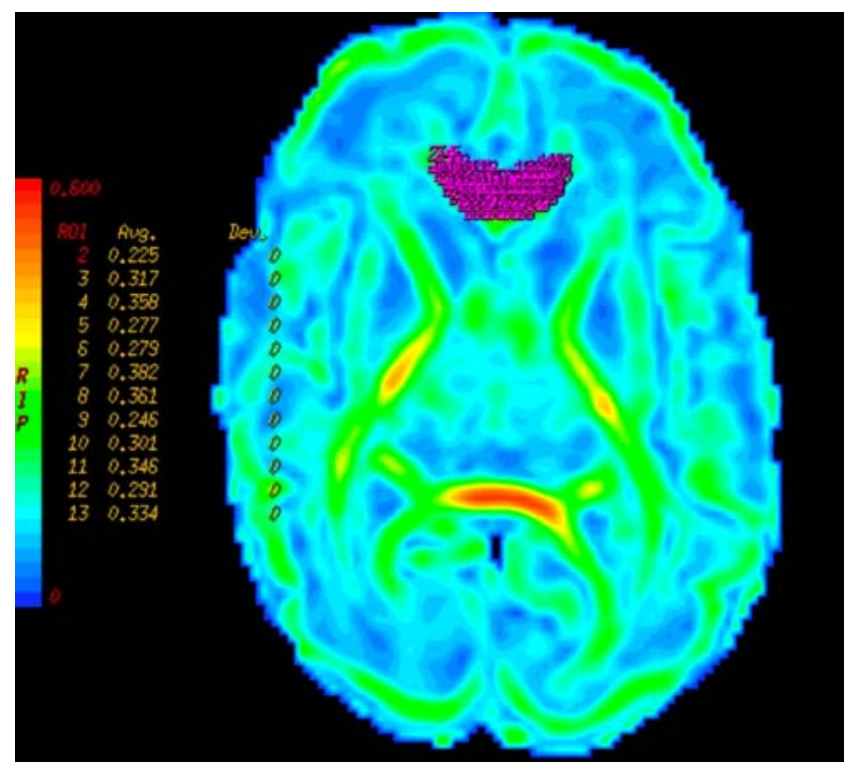

Fig. 5 Automated calculation of the maximum FA pixel value within the ROI on an FA map (gestational age 28 weeks) 
Fig. 6 Average FA values of 16-pixel ROIs of the PLIC

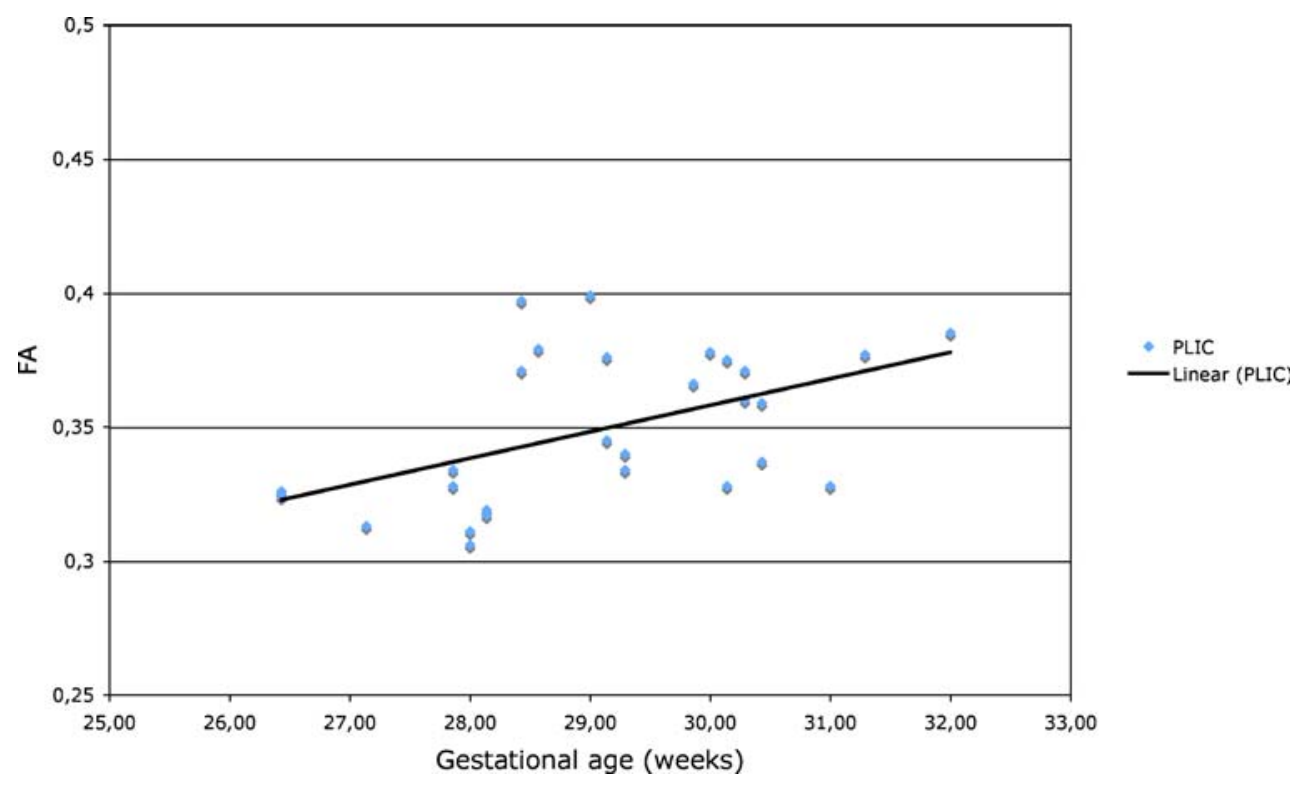

Comparison between tracts

DTI values varied between white-matter structures (Table 2). FA was highest in the commissural tracts of the corpus callosum (splenium $>$ genu) and deep projection tracts (internal capsule). FA was lowest in association tracts (external capsule).

\section{Discussion}

Our aim was to provide clinicians with reference values for the evaluation of diffusion tensor images of white-matter tracts in VLBW infants. A literature search identified four similar studies. Comparison with these studies seems irrelevant as each included no more than four infants evaluated below a gestational age of 32 weeks within the first days of life. We were able to study scans of 28 VLBW infants without white-matter damage within the first 4 days of life. We found a statistically significant correlation between gestational age and FA of the PLIC. Differences were observed between white-matter tracts: FA was highest in commissural, followed by deep projection and association tracts. Others have found the same hierarchy in the perinatal brain [16] and in fully myelinated adult tracts [27].

Different anisotropy indexes can be used to study the anisotropy of white-matter tracts: FA, relative anisotropy (RA), axial anisotropy etc. We focused on FA, the most frequently used anisotropy index. FA has a high sensitivity for studying anisotropy in white-matter tracts [16]; it has a higher SNR than RA for anisotropic regions [28, 29]. Previous studies have shown that FA is the most sensitive and significant discriminating DTI parameter in pair-wise comparisons between different tracts in premature infants [16]. In most tracts FA increases significantly by the week, whereas weekly decreases in ADC values are smaller and less discriminative in most regions [16]. There are a growing number of reports of FA values of injured tracts [30-32]. Nevertheless, we should realize that FA is only one of the functions that describe the diffusion tensor.
Table 2 Tract statistics (FA and ADC mean values and standard deviations) and significant differences in FA and ADC between tracts

\begin{tabular}{|c|c|c|c|c|}
\hline \multirow[t]{2}{*}{ Structure } & \multicolumn{2}{|l|}{ FA } & \multicolumn{2}{|l|}{$\mathrm{ADC}$} \\
\hline & Mean (SD) & $\begin{array}{l}\text { Tracts showing } \\
\text { significant } \\
\text { differences } \\
(P<0.05)\end{array}$ & Mean (SD) & $\begin{array}{l}\text { Tracts showing } \\
\text { significant } \\
\text { differences } \\
(P<0.05)\end{array}$ \\
\hline Posterior limb of internal capsule & $0.349(0.028)$ & $a, b, c$ & $1.09(0.05)$ & $1, \mathrm{~m}, \mathrm{n}, \mathrm{o}, \mathrm{p}$ \\
\hline Anterior limb of internal capsule & $0.242(0.033)$ & $a, d, e, f$ & $1.27(0.091)$ & 1 \\
\hline External capsule & $0.175(0.188)$ & $\mathrm{b}, \mathrm{g}, \mathrm{h}, \mathrm{i}$ & $1.29(0.097)$ & $\mathrm{m}$ \\
\hline Optic radiation & $0.270(0.042)$ & $\mathrm{g}, \mathrm{j}, \mathrm{k}$ & $1.30(0.126)$ & $\mathrm{n}$ \\
\hline Corpus callosum (genu) & $0.42(0.048)$ & $\mathrm{d}, \mathrm{e}, \mathrm{h}, \mathrm{j}$ & $1.24(0.111)$ & o \\
\hline Corpus callosum (splenium) & $0.442(0.056)$ & $\mathrm{c}, \mathrm{f}, \mathrm{i}, \mathrm{k}$ & $1.27(0.131)$ & $\mathrm{p}$ \\
\hline
\end{tabular}


Fig. 7 Maximum FA ROI values of the PLIC

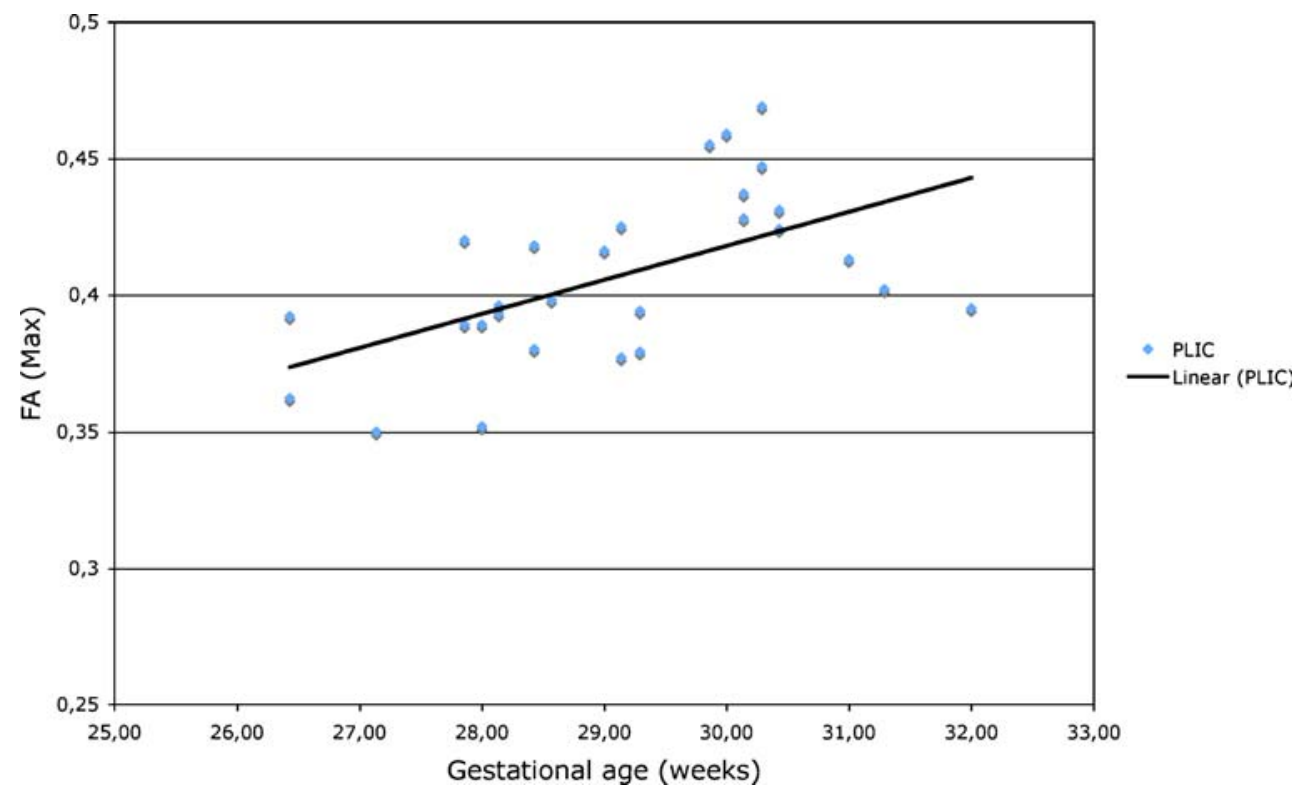

Although aberrant FA values seem to be a sensitive detector of abnormal tissue one could also use the relative magnitudes of the three principal eigenvectors to describe diffusion.

What determines anisotropy in white-matter tracts? The value of the anisotropic index is determined by size, shape and composition of physical obstructions, as well as the space between them. Myelin, fast axonal transport, the axonal cytoskeleton (neurofilaments and microtubules), and local susceptibility gradients do not seem to have any major impact on anisotropic water diffusion [26, 33]. Indeed, intact membranes are thought to be the main contributing factor to anisotropy. The increasing anisotropy of white matter takes place before the histological appearance of myelin [15]. This increase has been attributed to the 'premyelinating state' $[13,34]$. The corpus callosum, with a coherent parallel organization, has the highest FA values. Callosal fibre tracts, however, do not show a significant increase in FA with increasing gestational age, as the PLIC does. This might be due to the fact that myelination progresses more slowly in the callosal fibres than in the projection fibres at this age.

During development a decrease in ADC values of the white-matter tracts is expected. We found no correlation between average ADC values and gestational age in the studied tracts. When looking at the available data reported previously we only saw the ADC values decrease significantly with gestational age when the ADC values of the infants older then 35 weeks were included. The fact that we could not find any significant correlation could be due to the limited number of infants scanned. The alternative is that at an earlier gestational age there is no significant decrease in ADC in the studied tracts.
Studying DTI parameters of the white-matter tracts of VLBW infants is challenging for many reasons. One of these challenges is to determine a standard for the size and shape of the ROIs. A ROI is a controlled identification of a given area of an image for numerical analysis and the area of anatomy being scanned that is of particular importance in the image. Different authors have used different ways to set their ROIs. The reason we compared two techniques was to achieve better reproducibility. Maximum values (the maximum pixel value within a ROI) might be an alternative in which the value is given by the software. However, theoretically there is a bigger change of artefacts. Fibre tracking, colour maps and ADC maps are established but time-consuming techniques for the verification of tracts. We trust that automatic verification will become common practice in the future. A possible solution for ROI comparisons between researchers is the use of a neonatal brain atlas coordinate system. Individual brain images could then be transformed into a common coordinate space and the ROIs could be placed at specific topographic coordinates. Our research group is currently looking into this option.

Another serious challenge is the SNR and spatial resolution constraints due to the very low anisotropy of premyelinating white matter and the tiny size of white-matter tracts in premature newborns. Using a custom-made MRcompatible incubator with a high-sensitivity neonatal head coil that improved image quality, spatial resolution and patient comfort, we were able to overcome this challenge.

In this retrospective study a b value of $1,000 \mathrm{~s} / \mathrm{mm}^{2}$ was used to allow comparisons between early and later scans at different ages. We realize a lower $b$ value would be optimal because the ADC value in neonatal white matter is higher 
than that of more mature brains. The optimal $b$ value can be calculated as indicated by Jones et al. [35].

Like all VLBW infants discharged from our NICU, our study patients were routinely seen by trained paediatricians and by paediatric physical therapists at the outpatient department for neurodevelopmental follow-up. Neurodevelopmental outcome was defined according to the most recent neurological examination and Denver and Bayley scores. The children's ages at the most recent assessments varied, making the results difficult to compare; also Denver and Bayley scores are limited in their prognostic value below the age of 2 years.

Better understanding of normal preterm white matter development is essential to encourage the use of DTI for evaluation and treatment of white-matter injury. Early diagnosis of white-matter abnormalities means that early intervention might be possible. We are exploring the feasibility of perinatal brain repair, and new MR imaging techniques such as DTI will enable us to improve our understanding of how the developing brain responds to our interventions.

\section{Conclusion}

Our study gives anisotropy values for VLBW infants with normal outcome that can be used as reference values. This work adds to our understanding of normal preterm whitematter development.

Acknowledgement This study was supported by a grant from the Beatrix Foundation.

\section{References}

1. Costeloe K, Hennessy E, Gibson AT et al (2000) The EPICure study: outcomes to discharge from hospital for infants born at the threshold of viability. Pediatrics 106:659-671

2. Piecuch RE, Leonard CH, Cooper BA et al (1997) Outcome of extremely low birth weight infants (500 to 999 grams) over a 12-year period. Pediatrics 100:633-639

3. Wood NS, Marlow N, Costeloe K et al (2000) Neurologic and developmental disability after extremely preterm birth. N Engl J Med 343:378-384

4. Hack M, Taylor HG, Klein N et al (2000) Functional limitations and special health care needs of 10- to 14-year-old children weighing less than 750 grams at birth. Pediatrics 106:554-560

5. Taylor HG, Klein N, Hack M (2000) School-age consequences of birth weight less than $750 \mathrm{~g}$ : a review and update. Dev Neuropsychol 17:289-321

6. Barnes PD (2001) Neuroimaging and the timing of fetal and neonatal brain injury. J Perinatol 21:44-60

7. Volpe JJ (1997) Brain injury in the premature infant. Clin Perinatol 24:567-587

8. Huppi PS, Inder TE (2001) Magnetic resonance techniques in the evaluation of the perinatal brain: recent advances and future directions. Semin Neonatol 6:195-210
9. Robertson NJ, Wyatt JS (2004) The magnetic resonance revolution in brain imaging: impact on neonatal intensive care. Arch Dis Child Fetal Neonatal Ed 89:193-197

10. Rademaker KJ, Uiterwaal CS, Beek FJ et al (2005) Neonatal cranial ultrasound versus MRI and neurodevelopmental outcome at school age in children born preterm. Arch Dis Child Fetal Neonatal Ed 90:489-493

11. Yakovlev PI, Lecours AR (1967) The myelogenetic cycles of regional maturation of the brain. In: Mankowski A (ed) Regional development of the brain in early life. Blackwell, Oxford, pp 3-69

12. Sie LT, van der Knaap MS, van Wezeel-Meijler G et al (1997) MRI assessment of myelination of motor and sensory pathways in the brain of preterm and term born infants. Neuropediatrics 28:97-105

13. Wimberger DM, Roberts TP, Barkovich AJ et al (1995) Identification of "premyelination" by diffusion-weighted MRI. J Comput Assist Tomogr 19:28-33

14. Berman JI, Mukherjee P, Partridge SC et al (2005) Quantitative diffusion tensor MRI fiber tractography of sensorimotor white matter development in premature infants. Neuroimage 27:862-871

15. Huppi PS, Maier SE, Peled S et al (1998) Microstructural development of human newborn cerebral white matter assessed in vivo by diffusion tensor magnetic resonance imaging. Pediatr Res 44:584-590

16. Partridge SC, Mukherjee P, Berman JI et al (2005) Tractographybased quantitation of diffusion tensor imaging parameters in white matter tracts of preterm newborns. J Magn Reson Imaging 22:467-474

17. Yoo SS, Park HJ, Soul JS et al (2005) In vivo visualization of white matter fiber tracts of preterm- and term-infant brains with diffusion tensor magnetic resonance imaging. Invest Radiol 40:110-115

18. Frankenburg WK, Camp BW, van Natta PA (1971) Validity of the Denver developmental screening test. Child Dev 42:475-485

19. Bayley N (1993) Bayley scales of infant development, 2nd edn. Psychological Corporation, San Antonio, TX

20. Shellock FG, Crues JV (2004) MR procedures: biologic effects, safety, and patient care. Radiology 232:635-652

21. Groenendaal F, Leusink C, Nijenhuis M et al (2002) Neonatal life support during magnetic resonance imaging. J Med Eng Technol 26:71-74

22. Battin M, Maalouf EF, Counsell S et al (1998) Physiological stability of preterm infants during magnetic resonance imaging. Early Hum Dev 52:101-110

23. Basser PJ, Pierpaoli C (1996) Microstructural and physiological features of tissues elucidated by quantitative-diffusion-tensor MRI. J Magn Reson B 111:209-219

24. Pierpaoli C, Basser PJ (1996) Toward a quantitative assessment of diffusion anisotropy. Magn Reson Med 36:893-906

25. Le Bihan D, Mangin JF, Poupon C et al (2001) Diffusion tensor imaging: concepts and applications. J Magn Reson Imaging 13:534-546

26. Wakana S, Jiang H, Nagae-Poetscher LM et al (2004) Fiber tractbased atlas of human white matter anatomy. Radiology 230:77-87

27. Shimony JS, McKinstry RC, Akbudak E et al (1999) Quantitative diffusion-tensor anisotropy brain MR imaging: normative human data and anatomic analysis. Radiology 212:770-784

28. Hasan KM, Alexander AL, Narayana PA (2004) Does fractional anisotropy have better noise immunity characteristics than relative anisotropy in diffusion tensor MRI? An analytical approach. Magn Reson Med 51:413-417

29. Van Pul C, Buijs J, Janssen MJ et al (2005) Selecting the best index for following the temporal evolution of apparent diffusion coefficient and diffusion anisotropy after hypoxic-ischemic white matter injury in neonates. AJNR 26:469-481

30. Counsell SJ, Allsop JM, Harrison MC et al (2003) Diffusionweighted imaging of the brain in preterm infants with focal and diffuse white matter abnormality. Pediatrics 112:1-7 
31. Huppi PS, Murphy B, Maier SE et al (2001) Microstructural brain development after perinatal cerebral white matter injury assessed by diffusion tensor magnetic resonance imaging. Pediatrics 107:455-460

32. Nagy Z, Westerberg H, Skare S et al (2003) Preterm children have disturbances of white matter at 11 years of age as shown by diffusion tensor imaging. Pediatr Res 54:672-679
33. Beaulieu C (2002) The basis of anisotropic water diffusion in the nervous system: a technical review. NMR Biomed 15:435-455

34. Huppi PS, Dubois J (2006) Diffusion tensor imaging of brain development. Semin Fetal Neonatal Med 11:489-497

35. Jones DK, Horsfield MA, Simmons A (1999) Optimal strategies for measuring diffusion in anisotropic systems by magnetic resonance imaging. Magn Reson Med 42:117-121 